\title{
A Protein Extraction System with a Water/Oil Microemulsion formed by a Biodegradable Polymer Surfactant
}

\author{
Satoru NISHINO ${ }^{1,2}$, Akio KISHIDA ${ }^{3}$, Hidekazu YOSHIZAWA ${ }^{2}$, and Koichiro SHIOMORI ${ }^{4} *$ \\ ${ }^{1}$ Department of Chemical Engineering, Nara National College of Technology, Nara 639-1080 Japan \\ ${ }^{2}$ Department of Material \& Energy Science, Graduate School of Environmental Science, \\ Okayama University, Okayama 700-8530, Japan \\ ${ }^{3}$ Institute of Biomaterials and Bioengineering, Tokyo Medical and Dental University, \\ Tokyo 101-0062, Japan \\ ${ }^{4}$ Department of Applied Chemistry, University of Miyazaki, Miyazaki 889-2192, Japan \\ (Received May 13, 2013; Accepted July 30, 2013)
}

The amphiphilic biodegradable polymer surfactant, poly(ethyleneoxide monooleate)-blockpoly $(D, L$-lactide) (MOPEO-PLA), was shown to form microemulsions in chloroform solution and to extract water and protein into their microemulsions from the aqueous phase. The water content in the chloroform phase significantly increased with the MOPEO-PLA concentration, indicating that a MOPEO-PLA microemulsion was formed with a large amount of water molecules. The largest amount of cytochrome $c$ in the MOPEO-PLA microemulsion phase was extracted when the $\mathrm{pH}$ of the aqueous phase was close to the $\mathrm{pI}$ value for cytochrome $c$, and the degree of extraction increased at higher temperatures. From these results, the main factor for the extraction of cytochrome $c$ was found to be the hydrophobic interaction between MOPEO-PLA and cytochrome $c$. The MOPEO-PLA microemulsion provides a benign extraction process for proteins. A MOPEO-PLA microemulsion system would provide a new encapsulation system in a biodegradable polymer matrix for delivery of proteins and peptides.

\section{Introduction}

Recently, microencapsulation techniques for water-soluble bioactive agents such as proteins, peptides, nucleic acids, and human growth hormones, which have short half-lives in vivo and are unstable in biological fluids, have been developed for the purpose of long-term sustained-release formulations [1-3]. Biodegradable polymer microcapsules enclosing bioactive agents had mostly been produced by a solvent evaporation method via a double emulsion system, which efficiently traps hydrophilic agents in microcapsules by trapping an inner aqueous phase with the agents in the oil phase compartments $[4,5]$. However, double emulsions are thermodynamically unstable due to coalescence and the large size distribution of the inner aqueous phase; therefore protein-loaded microcapsules release protein uncontrollably [6]. Furthermore, it has been demonstrated that sensitive proteins lost their activity at the W/O interface [7, 8], and produced a denatured protein through shear-induced stress with homogenization when an inner aqueous phase was dispersed in the oil phase [9].

W/O microemulsions, which are self-assemblages of amphiphilic molecules in a nonpolar organic 
solvent, can solubilize hydrophilic macromolecules and bioactive agents in their micro water pool [10-14]. Therefore, W/O microemulsions solubilizing hydrophilic bioactive agents are uniform and thermodynamically stable. A bioactive agent can be uniformly distributed in a microcapsule by adapting bioactive agents solubilized in a uniform W/O microemulsion to the $\mathrm{O} / \mathrm{W}$ emulsion system. Furthermore, it is considered that this microencapsulation system is benign for bioactive agents because the microemulsion solubilized bioactive agents form spontaneously without intensive agitation. Hayashi et al. reported on microencapsulation of bioactive agents using a W/O microemulsion system using di-2-ethylhexyl sodium sulfosuccinate (AOT) and a sucrose ester of fatty acids [15]. However, these surfactants were not suitable because of their immiscibility with the polymer matrix of the microcapsules such as poly(lactide); (PLA) and poly(lactide-co-glycolide).

We designed a hydrophobic biodegradable polymer surfactant, poly(ethyleneoxide monooleate)-block-poly(D,L-lactide); (MOPEO-PLA), whose chemical structure is shown in Figure 1, for the use with accurate-release polymer microcapsules [16]. MOPEO-PLA can form a W/O microemulsion in the organic phase, which solubilizes hydrophilic bioactive agents, and which has good miscibility with the matrix of the PLA microcapsules. In the present study, we investigated the formation of MOPEO-PLA microemulsions and the extraction of cytochrome $c$, as a model protein, by the microemulsion. As far as we know, this is the first report of protein extraction with a PLA-based polymeric surfactant system. To investigate the extraction behavior of cytochrome $c$, the liquid-liquid extraction was performed under varying operating conditions such as $\mathrm{pH}$, buffer

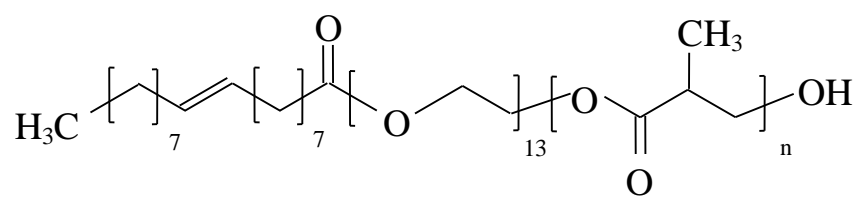

Figure 1. Molecular structure of MOPEO-PLA block copolymers.

concentration, and temperature.

\section{Experimental}

\subsection{Synthesis of MOPEO-PLA}

D,L-lactide was purified by recrystallization from toluene and then dried for over $24 \mathrm{hr}$ in vacuo at room temperature. Poly(ethyleneoxide monooleate) $(M w: 1,112)$ was purchased from Aldrich and used without further purification. Cytochrome $c$ from horse heart was purchased from Sigma. Other reagents were purchased from Wako Pure Chemical Industries, Ltd. MOPEO-PLA was synthesized by bulk ring-opening polymerization of D,L-lactide initiated with MOPEO in the presence of a stannous 2-ethylhexanoate catalyst according to the procedure given in our previous report [16]. The polymerization was carried out in a sealed glass ampoule at $403 \mathrm{~K}$ for 24 hours. After this time, the product was dissolved in chloroform and precipitated these from by addition of an excess amount of hexane. Finally, MOPEO-PLA was isolated and dried at $323 \mathrm{~K}$ under vacuum. The obtained polymer showed a single peak in the GPC trace and a polydispersity of less than 1.4 [16]. The molecular weight of MOPEO-PLA used in this study was about $2.0 \times 10^{3}-7.0 \times 10^{3}$.

\subsection{Extraction of water and cytochrome $c$}

The extraction of water in the organic phase was carried out by mixing a $\mathrm{NaCl}$ aqueous solution or distilled water with an equal volume of the MOPEO-PLA organic phase. In the water extraction 
experiments, the $\mathrm{pH}$ of the aqueous phase was not controlled. The concentration of cytochrome $c$ in the initial aqueous phase was fixed at $0.2 \mathrm{mg} / \mathrm{ml}$ and the buffer solutions were phthalic acid buffer ( $\mathrm{pH} 4.0$ ), phosphate buffer ( $\mathrm{pH}$ 7.2), and boric acid buffer ( $\mathrm{pH}$ 9.2). The organic phase was a chloroform solution of MOPEO-PLA and $20 \mathrm{wt} \%$ of methanol as a co-solvent. Methanol is the most effective co-solvent for the extraction of cytochrome $c$ among alcohols, such as methanol, ethanol, $n$-propanol, isopropanol, $n$-butanol and hexanol. The extraction of cytochrome $c$ into the organic phase was carried out by mixing an aqueous buffer solution containing cytochrome $c$ with an equal volume of the MOPEO-PLA organic phase. After mixing the two phases by shaking for $24 \mathrm{hr}$ at $298 \mathrm{~K}$, the clear organic phase was collected by centrifugation at 6,000 rpm for $10 \mathrm{~min}$. The water content in the organic phase was measured with a Karl-Fischer titrator (Kyoto Electronics, MKA-3), and the concentrations of cytochrome $c$ in the organic and in the aqueous phases were determined by measuring the absorbance at $408 \mathrm{~nm}$ using a UV-VIS spectrophotometer (Hitachi, U-2000A). The molar ratio of water to surfactant, $W o=\left[\mathrm{H}_{2} \mathrm{O}\right] /[\mathrm{MOPEO}-\mathrm{PLA}]$ was used to characterize the microemulsions.

\section{Results and Discussion}

\subsection{Extraction properties of water in an organic phase containing MOPEO-PLA}

The effects of the MOPEO-PLA concentration on the water content in the organic phase are shown in Figure 2. The water content significantly increased at MOPEO-PLA concentrations higher than $10 \mathrm{mM}$. It was therefore considered that a MOPEO-PLA microemulsion solubilizing a large amount of water in the organic phase was formed. The water content was slightly higher when the MOPEO-PLA molecule had a longer hydrophobic PLA segment. This was due to an increase in the number of MOPEO-PLA microemulsions, because the MOPEO-PLA with the longer PLA segments formed a MOPEO-PLA microemulsion with a smaller number of MOPEO-PLA molecules which cause an increase in the total number of the microemulsions. Information of the size of the microemulsions is important in any discussion of these results. However, the diameter of the MOPEO-PLA microemulsion could not be observed by using a dynamic light scattering method because of the lack of scatter intensity from the polymer colloid solution. To investigate the detailed characteristics of the MOPEO-PLA microemulsion system, another method such as small angle neutron scattering should be used in future work.

The effect of the $\mathrm{NaCl}$ concentration on the water content in the organic phase for MOPEO-PLA concentrations of 10 and $20 \mathrm{mM}$, at which a MOPEO-PLA microemulsion was formed as shown in Figure 2, is shown in Figure 3. It is clear that the water content was little affected by the $\mathrm{NaCl}$ concentration, in contrast to the effect of

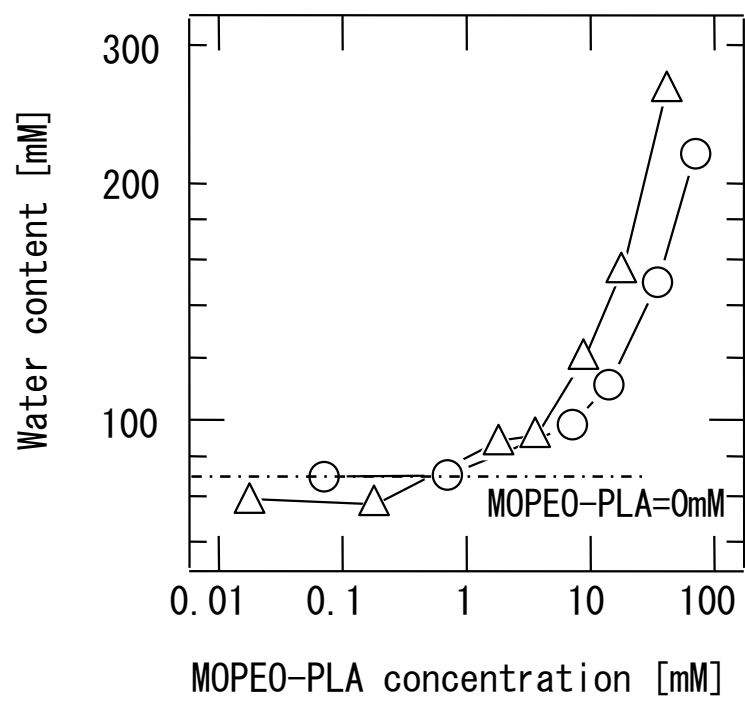

Figure 2. Effect of MOPEO-PLA concentration on the water content in the chloroform phase. $M w$ of MOPEO-PLA was $1.8 \times 10^{3}(O)$ and $6.5 \times 10^{3}(\triangle)$. Distilled water was used as the aqueous phase. 
the $\mathrm{NaCl}$ concentration with a conventional microemulsion system using an ionic surfactant such as AOT, because MOPEO-PLA is a nonionic polymer surfactant. The decreased amount of water caused by an increase in the $\mathrm{NaCl}$ concentration at $10 \mathrm{mM}$ MOPEO-PLA was larger than that at 20 $\mathrm{mM}$. As a result, the values of Wo, which is commonly used to characterize microemulsions, were affected by the concentration of MOPEO-PLA. In a conventional microemulsion system using AOT, the Wo values are unaffected by the AOT concentration. These tendencies were quite different from the result of the previous experiment with AOT [17]. It was thus inferred that the MOPEO-PLA microemulsion was self-organized in a state of ambiguous aggregation with polymeric PEO segments and a quite large hydrophobic PLA chain unlike the AOT microemulsion, which would form tight spherical aggregates. Further investigation on the structure of the MOPEO-PLA microemulsion is required.

\subsection{Extraction properties of cytochrome $c$ with the MOPEO-PLA microemulsion system}

The extraction of cytochrome $c$ was carried out using the organic phase containing MOPEO-PLA. The effect of the MOPEO-PLA concentration on the extraction percentage of cytochrome $c$ is shown in Figure 4. The extraction percentage increased above a MOPEO-PLA concentration of $10 \mathrm{mM}$. This concentration corresponded to the water content result shown in Figure 2, indicating that cytochrome $c$ in the aqueous phase was solubilized in the organic phase by forming a complex with the MOPEO-PLA microemulsion.

The visible adsorption spectra of cytochrome $c$ extracted into the MOPEO-PLA microemulsion solution and native cytochrome $c$ dissolved in the buffer solution are illustrated in Figure 5. An adsorption peak at $408 \mathrm{~nm}$ was observed in both spectra, and indicated that cytochrome $c$ was extracted in the MOPEO-PLA microemulsion. In addition, the adsorption at near $530 \mathrm{~nm}$, assigned to the heme of cytochrome $c$ was also similar to that for unextracted cytochrome $c$, suggesting that the conformation of heme of cytochrome $c$ extracted in the

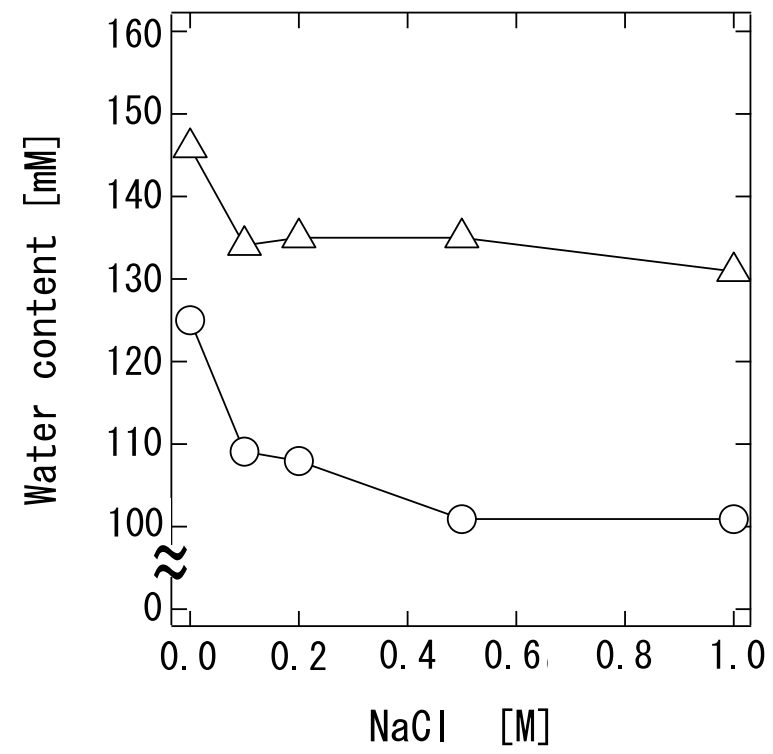

Figure 3. Effect of $\mathrm{NaCl}$ concentration on the water content of the organic phase. The MOPEO-PLA concentration was $(\mathrm{O}) 10 \mathrm{mM}$ and $(\triangle) 20 \mathrm{mM} . \mathrm{Mw}$ of MOPEO-PLA was $4.0 \times 10^{3}$.

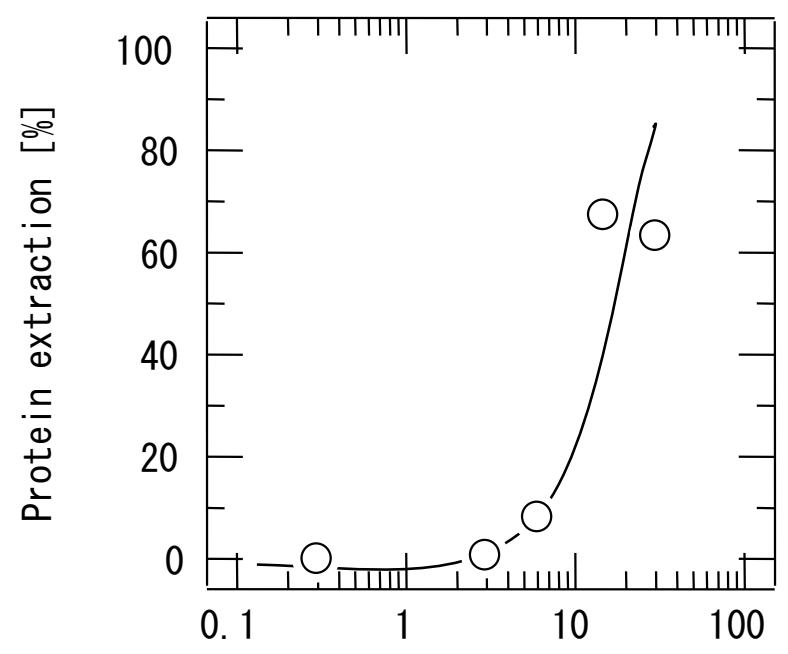

MOPE0-PLA concentration [mM]

Figure 4. Effect of the MOPEO-PLA concentration on the extraction percentage of cytochrome $c$. The $M w$ of MOPEO-PLA was $4.4 \times 10^{3}$ and the $\mathrm{pH}$ was 7.2. 
MOPEO-PLA microemulsion was not appreciably altered.

The effect of $\mathrm{pH}$ on the extraction percentage of cytochrome $c$ is shown in Figure 6. The maximum extraction percentage was at $\mathrm{pH} 10.1$, which corresponds to the $\mathrm{p} I$ value for cytochrome $c$. It was, therefore, considered that there was no electrostatic interaction between the protein and the surfactant because the net charge of the protein is almost zero when the $\mathrm{pH}$ of the aqueous phase is equal to $\mathrm{p} I$. Furthermore, the $\mathrm{pH}$ of the aqueous phase strongly influenced the extraction behavior of cytochrome $c$. This tendency was due to the cytochrome $c$ being more stable in the MOPEO-PLA microemulsion than in the aqueous phase because the solubility of the protein was the lowest at the $\mathrm{p} I$ value of the protein.

Figure 7 shows the effect of the boric buffer concentration on the extraction behavior of

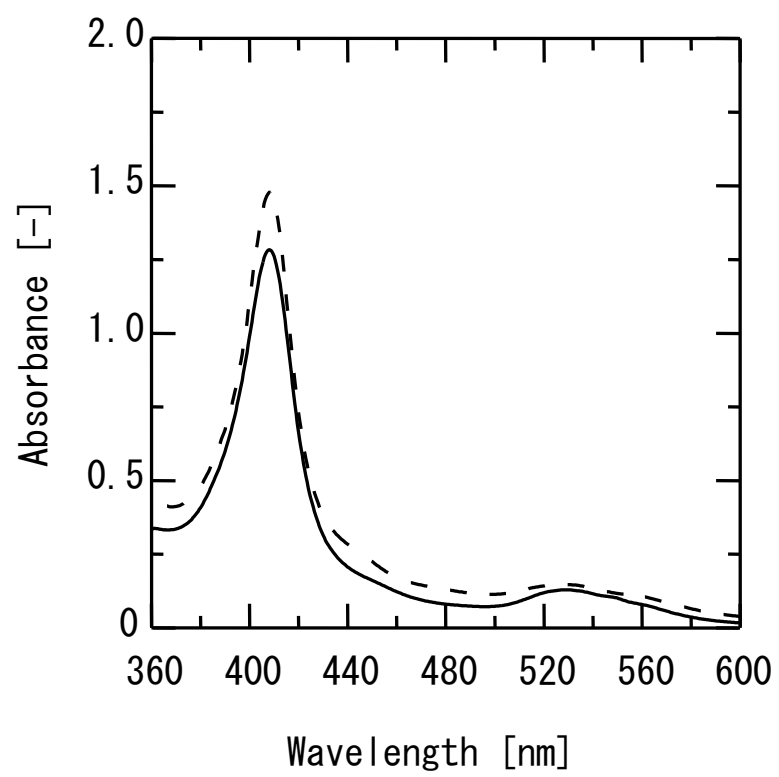

Figure 5. Absorption spectra of cytochrome $c$ extracted into the MOPEO-PLA microemulsion (broken line) and native cytochrome $c$ in the aqueous phase (solid line). cytochrome $c$ when the $\mathrm{pH}$ of the aqueous phase was adjusted to the $\mathrm{p} I$ value. The protein extraction gradually decreased with an increase in the buffer concentration. However, the decrease, which was generally caused by the shielding of the electrostatic interaction between the protein and the microemulsion, is not so large as compared with that of other microemulsion systems [20]. This result also confirmed the pH dependency shown in Figure 6 whereby the electrostatic interaction between the protein and the MOPEO-PLA microemulsion was not the dominant driving force for the extraction of cytochrome.

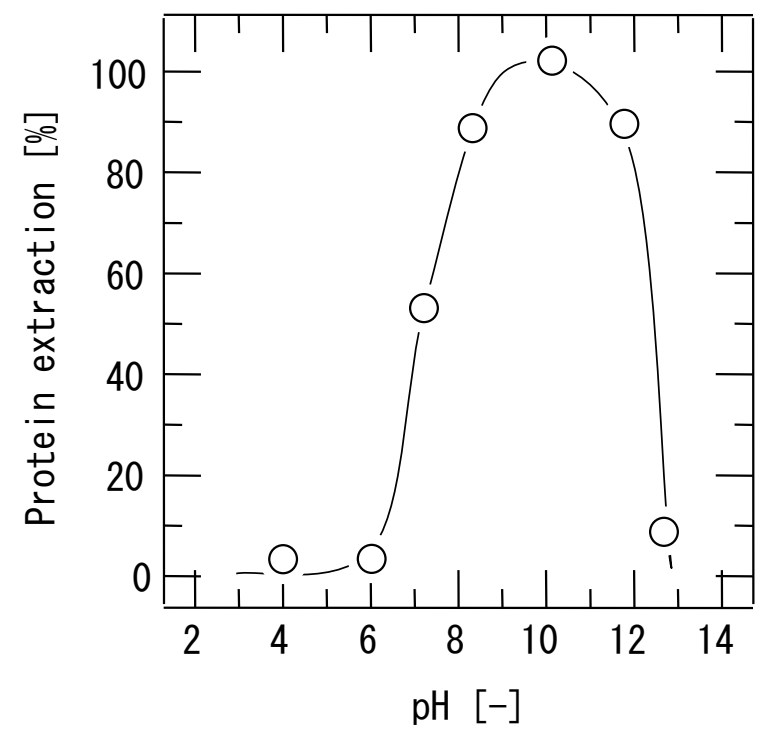

Figure 6. Effect of $\mathrm{pH}$ on the extraction percentage of cytochrome $c$. The $M w$ of MOPEO-PLA was $4.4 \times 10^{3}$ and the concentration of MOPEO-PLA was $33.9 \mathrm{mM}$.

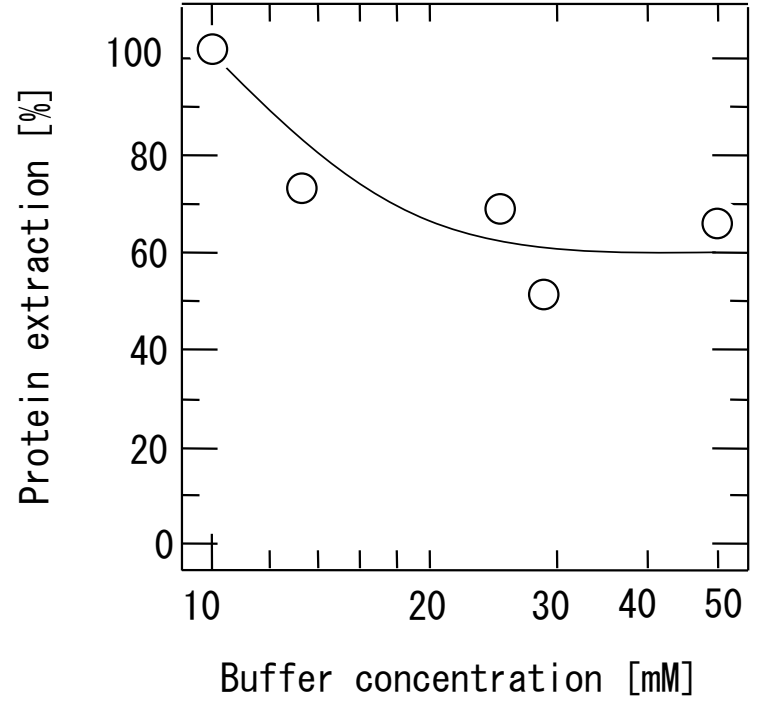

Figure 7. Effect of boric buffer concentration on the extraction percentage of cytochrome $c$. The $M w$ of MOPEO-PLA was $4,1 \times 10^{3}$. the $\mathrm{pH}$ was 10.1 , and the concentration of MOPEO-PLA was $36.1 \mathrm{mM}$. 
The effect of $\mathrm{pH}$ and ionic strength in the aqueous media in an ionic AOT reveres micelle system shows that the driving force for the formation of a protein-surfactant complex in the organic phase is mainly governed by the electrostatic interaction between the charge group of the surfactant and the protein $[18,19]$. It was also reported that the protein was extracted by electrostatic interaction with the nonionic surfactant [20]. In this study, the protein was considered to be extracted in a MOPEO-PLA microemulsion through different interactions than the electrostatic interaction. It is supposed that the intermolecular force between the protein and the MOPEO-PLA microemulsion is hydrophobic or a hydrogen bond. The strength of these interactions is strongly dependent on temperature. Pires and Cabral reported that cytochrome $b_{5}$ was effectively extracted with an ionic surfactant through a hydrophobic interaction at a $\mathrm{pH}$ value close to the $\mathrm{p} I$ value, and that the extraction performance strongly depended on the temperature at the cytochrome $b_{5} \mathrm{p} I$ value[21].

The temperature dependency was investigated to clarify the extraction mechanism of cytochrome $c$ with MOPEO-PLA microemulsions in the organic phase. The effect of temperature on the extraction percentage of cytochrome $c$ is shown in Figure 8. The extraction percentage increased with temperature. The water molecules in the MOPEO-PLA microemulsion were held by a hydrogen bond with the ether bond of poly(ethylene oxide). The hydrophobicity in the MOPEO-PLA microemulsion increased with increasing temperature because of the exclusion of the hydrated water molecule in the MOPEO-PLA microemulsion by high molecular mobility at the higher temperature. The temperature dependency indicates that hydrophobic interaction governed the MOPEO-PLA microemulsion system's ability to extract cytochrome $c$.

The effect of the molecular weight of MOPEO-PLA on the extraction behavior of cytochrome $c$ and water in the organic solution is shown in Figure 9.

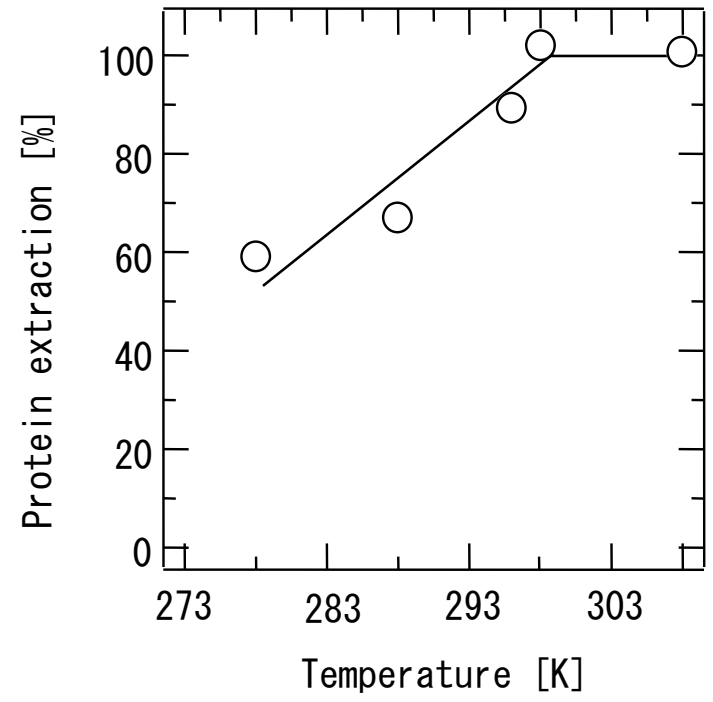

Figure 8. Effect of temperature on the extraction percentage of cytochrome $c$. The $M w$ of MOPEO-PLA was $4.1 \times 10^{3}$, the $\mathrm{pH}$ was 10.1 , and the concentration of MOPEO-PLA was $36.1 \mathrm{mM}$.

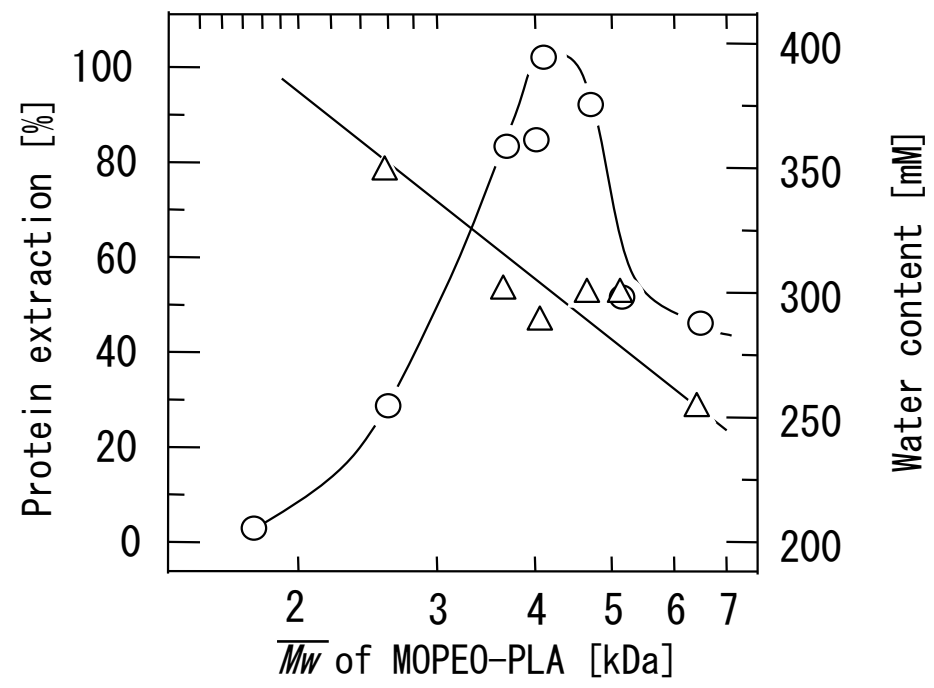

Figure 9. Effect of the MOPEO-PLA molecular weight on the extraction percentage of cytochrome $c(\bigcirc)$ and the water content in the organic phase $(\triangle)$. The $\mathrm{pH}$ was 10.1 , and the concentration of MOPEO-PLA was $10 \mathrm{wt} \%$. 
When the molecular weight of MOPEO-PLA was less than $4 \times 10^{3}$, the extraction percentage of cytochrome $c$ significantly increased with increasing molecular weight of MOPEO-PLA despite the fact that the water content in the organic phase decreased. The increase in the molecular weight of MOPEO-PLA was caused by the increase in the hydrophobic PLA chain. So, the complex between cytochrome $c$ and the MOPEO-PLA microemulsion would become more hydrophobic by the increase in the PLA chain and extraction of cytochrome $c$ increased. In contrast, a higher molecular weight of the MOPEO-PLA caused the extraction percentage of cytochrome $c$ to decrease. This may be because a bulky MOPEO-PLA microemulsion with longer PLA segments has difficulty in entrapping cytochrome $c$ into the water pool of the microemulsion because of steric hindrance. In this way, the MOPEO-PLA microemulsion with suitable properties efficiently extracted cytochrome $c$ through the hydrophobic interaction. It is considered that this extraction process is benign for proteins because the hydrophobic force between the cytochrome $c$ and the MOPEO-PLA is weak compared with electrostatic interaction. In other research in which we analyzed the initial reduction rate coefficient of cytochrome $c$ reduced with ascorbic acid, cytochrome $c$ was microencapsulated with PLA using a MOPEO-PLA microemulsion system and the activity of cytochrome $c$ released from the microcapsules was kept at almost $100 \%$ [16].

\section{Conclusion}

The formation of a polymer microemulsion and protein extraction in organic media was investigated using MOPEO-PLA as a biodegradable polymer surfactant. It was found that a MOPEO-PLA microemulsion was formed by water solubilization in a MOPEO-PLA organic solution. An analysis of the visible spectra of the organic clear solution reveals that cytochrome $c$ was extracted into the MOPEO-PLA microemulsion system with methanol as a co-solvent. The maximum percentage of cytochrome $c$ extraction was obtained at $\mathrm{pH} 10.1$ equal to the $\mathrm{p} I$ value for cytochrome $c$. The effect of $\mathrm{pH}$, buffer concentration, operation temperature, and the $M w$ of MOPEO-PLA, depending on the hydrophobic PLA chain, on extraction ability indicated that the main factor for complex formation between cytochrome $c$ and MOPEO-PLA was the hydrophobic interaction. Therefore, this system can provide a benign environment for protein separation and purification processes.

\section{References}

1) P. Johansen, Y. Men, H. P. Merkle, B. Gander, Eur. J. Pharm. Biochem., 50, 129-146 (2000).

2) J. L. Cleland, R. Langer, ACS Symposium Series, 567, 1-21 (1994).

3) R. K. Gupta, M. Singh, D. T. O’Hagan, Adv. Drug. Del. Res., 32, 225-246 (1998).

4) Y. Kawano, K. Shiomori, S. Kiyoyama, K. Takeshita, Y. Hatate, J. Chem. Eng. Japan, 34, 1182-1186 (2001).

5) F. T. Meng, G. H. Ma, W. Qiu, Z. G. Su, J. Control. Rel., 91, 407-416 (2003).

6) G. D. Rosa, R. Iommelli, M. I. La Rotonda, A. Miro, F. Quaglia, J. Control. Rel., 69, 283-295 (2000).

7) P. Couvreur, M. J. Blanco-Prieto, F. Puisieux, B. Roques, E. Fattal, Adv. Drug Del. Rev., 28, 85-96 (1997).

8) H. Sah, J. Pharm. Sci., 88, 1320-1325 (1999).

9) M. Diwan, T. G. Park, J. Control. Rel., 73, 233-244 (2001). 
10) P. L. Luisi, P. Meier, V. E. Imre, A. Pande, Reverse Micelles, Plenum Press, 323-337 (1984).

11) E. B. Leodidis, T. A. Hatton, Structure and Reactivity in Reverse Micelles, Elsevier Co., 270-302 (1989).

12) K. Shiomori, Y. Kawano, R. Kuboi, I. Komasawa, J. Chem. Eng., Japan, 28, 803-809 (1995).

13) T. Kinugasa, Y. Miyauchi, C. Nakano, K. Itoh, Y. Nishii, Solvent Extr. Res. Dev., Jpn, 12, 159-167 (2005).

14) Y. Toyokawa, S. Tsukahara, T. Fujiwara, Solvent Extr. Res. Dev., Jpn, 20, 29-38 (2013).

15) Y. Hayashi, S. Yoshioka, Y. Aso, A. L. W. Po, T. Terao, Pharmaceutical Research, 11, 337-340 (1994).

16) S. Nishino, Y. Kitamura, A. Kishida, H. Yoshizawa, Macromol. Biosci., 5, 1066-1073 (2005).

17) S. Ichikawa, S. Furusaki, Food and Bioproducts Processing, 73, 33-39 (1995).

18) K. E. Goklen, T. A. Hatton, Sep. Sci. Technol., 22, 831-841 (1987).

19) K. Shiomori, Y. Kawano, R. Kuboi, I. Komasawa, J. Chem. Eng., Japan, 33, 800-804 (2000).

20) K. Naoe, M. Nishino, T. Ohsa, M. Kawagoe, M. Imai, J. Chemical Technology and Biotechnology, 74, 221-226 (1999).

21) M. J. Pires, J. M. S. Cabral, Biotechnol. Prog., 9, 647-650 (1993). 